COMMENT. MR imaging and intracranial ECoG recordings are essential determinants of outcome of patients with MCD and medically intractable epilepsy. Neuronal proliferation pathology carries a better prognosis than abnormalities of cortical migration or organization. Surgery can result in $67 \%$ long-term seizure freedom.

\title{
MEMORY DEFICITS IN CHILDREN WITH MESIAL TEMPORAL SCLEROSIS AND EPILEPSY
}

Researchers at Sao Paulo School of Medicine, Brazil, analyzed the occurrence of episodic memory (for personally experienced events) and semantic memory (for stored knowledge acquired in the past) deficits in 19 consecutive children with mesial temporal sclerosis (MTS) (8-16 years old; mean IQ 97). Patients performed worse on tests of immediate and delayed verbal episodic memory, visual episodic memory, verbal and visual learning, mental scanning for semantic clues, object naming, word definition, and repetition of sentences. Patients with a history of status epilepticus had worse visual episodic memory, whereas patients with uncontrolled daily and weekly seizures had worse verbal learning. Patients on polytherapy were more impaired in visual learning. Early age of seizure onset had a significant negative impact on semantic memory tests. Except for a lower score on the Boston Naming Test with left sided MTS cf right, episodic or semantic memory tests showed no differences with laterality of MTS. (Rzezak P, Guimaraes C, Fuentes D, Guerreiro MM, Valente KDR. Episodic and semantic memory in children with mesial temporal sclerosis. Epilepsy Behav July 2011;21:242-247). (Respond: Dr Patricia Rzezak, Rua Abdo Ambuba, 75/31 Sao PauloSP, Brazil, 05725-030. E-mail: patriciarzezak@gmail.com).

COMMENT. MTS epilepsy in children is associated with significant deficits in episodic and semantic memory function, despite normal intelligence. The earlier the onset of epilepsy, the more severe is the impairment of semantic memory. Definition of distinct domains of memory is required for rehabilitation measures.

Tests of everyday verbal memory in $\mathbf{1 3 2}$ children with epilepsy were predictive of academic performance but not significantly correlated with reports of prospective memory. (Chapieski L et al. Epilepsy Behav July 2011;21:285-290).

\section{ATTENTION DEFICIT DISORDERS}

\section{EFFICACY AND SAFETY OF METHYLPHENIDATE FOR ADHD AND UNCONTROLLED SEIZURES}

Twenty-four participants with uncontrolled epilepsy and ADHD in this prospective study, conducted at University of Joinville, Santa Catarina, Brazil, were 7 16 years of age. They had been followed for at least 6 months before introduction of methylphenidate (MPH), and had at least 2 seizures treated with antiepileptic drugs. MPH dose started at $5 \mathrm{mg}$ once or twice daily, and was increased by $10 \mathrm{mg}$ weekly as necessary, not exceeding $60 \mathrm{mg}$ daily. MPH was effective in control of ADHD in 70.8\% 
patients, no change in $20.8 \%$, and symptoms were worse in $8.3 \%$. Compared to the previous 6 months, seizures were increased in number in 2 children $(8.3 \%)(\mathrm{P}<0.001)$, and not increased in $22(91.6 \%) ; 7$ patients had fewer seizures; $58.3 \%$ had partial epilepsy and $41.7 \%$ generalized epilepsy. ADHD was inattentive type in $41.7 \%$, combined type in $37.5 \%$, and $20.8 \%$ hyperactive/impulsive type. Results of 11 previous reports of MPH, ADHD and epilepsy are summarized: 5 studies reported an increase in seizures and 6 showed no increase. One controlled study of OROS-MPH observed a daily increased risk of seizures with increasing doses of the stimulant. (Koneski JAS, Casella EB, Agertt F, Ferreira MG. Efficacy and safety of methylphenidate in treating ADHD symptoms in children and adolescents with uncontrolled seizures: A Brazillian sample study and literature review. Epilepsy Behav July 2011;21:228-232). (Respond: Dr Julio AS Koneski. E-mail: juliokoneski@neurologica.com.br).

COMMENT. The majority of studies reporting no or small risk of seizures with MPH and epilepsy involve patients whose seizures are controlled with AEDs. Few studies investigate the safety of MPH and other stimulants in treatment of ADHD in children with epilepsy or epileptiform EEG, not treated with AEDs.

In a group of 234 children with uncomplicated ADHD who received EEGs, 36 (15.4\%) showed epileptiform abnormalities, $40 \%$ rolandic spikes and $60 \%$ focal abnormalities. With introduction of stimulant therapy in $205(87.6 \%)$ patients, seizures occurred only in the stimulant-treated group: in one of $175(0.6 \%)$ patients with a normal EEG and in $3(10 \%)$ of 30 with epileptiform EEGs. Seizures occurred in 2 of $12(16.7 \%)$ with EEG rolandic spikes (Hemmer SA, Pasternak JF et al. Pediatr Neurol 2001;24(2):99-102). The authors suggest that an epileptiform EEG in neurologically normal children with ADHD predicts considerable risk for the occurrence of seizures during stimulant therapy whereas a normal EEG carries minimal and no increased risk.

\section{COMPUTER-ASSISTED MANAGEMENT OF ADHD}

Researchers at the Departments of Psychiatry and Pediatrics, Children's Memorial Hospital, Chicago, conducted a randomized medication trial for ADHD involving 24 pediatric practices. Patients were randomly assigned to 2 groups, treatment as usual or a specialized care group in which physicians received 2 hours of ADHD medication management training plus training on a software program to monitor response. Parent and teacher reports were obtained before treatment and 4, 9, and 12 months after starting medication. Exclusion criteria included IQ $<70$, history of intolerance to stimulants, and ADHD medication in previous 2 months. Of 270 children participating, 208 (77\%) were boys and $62(23 \%)$ girls, mean age 8.2 years. Comparisons between groups were made for the highest daily dose of medication, with stimulant medications converted to methylphenidate equivalents. Total daily medication dose did not differ significantly between groups: mean $27.1 \mathrm{mg} /$ day for titration group and $27.9 \mathrm{mg} /$ day for patients not adhering to dose titration. Children in both groups improved on the ADHD Rating Scales and SNAP-IV, with no group differences in rate of improvement. Starting on a shortacting versus long-acting stimulant showed no significant difference in benefit but significant effects were associated with progressing sequentially until symptoms were in the average range $(\mathrm{P}=0.039)$. Brief physician training alone was of no added benefit, but 